\title{
Avaliação da prevalência da sintomatologia de disfunção temporomandibular em universitários de Odontologia de uma instituição pública de ensino
}

\section{Evaluation of the prevalence of symptomatology of temporomandibular dysfunction in dentistry universities} of a public education institution

Evaluación de la prevalencia de sintomatología de la disfunción temporomandibular en universidades de odontología de una institución de educación pública

Amanda de Sá Barreto de FREITAS ${ }^{1}$

José Henrique de Araújo CRUZ1

Fausta Maria Moura de CASTRO1

Elizandra Penha da SILVA2

Abrahão Alves de OLIVEIRA FILHO²

Gymenna Maria Tenório GUÊNES²

${ }^{1}$ Curso de Graduação em Odontologia, Centro de Saúde e Tecnologia Rural, Universidade Federal de Campina Grande (UFCG) 58708-110 Patos - PB, Brasil

${ }^{2}$ Professor(a) Doutor(a), Curso de Graduação em Odontologia, Centro de Saúde e Tecnologia Rural, Universidade Federal de Campina Grande (UFCG) 58708-110 Patos - PB, Brasil

\section{Resumo}

Introdução: A disfunção temporomandibular é um conjunto de condições dolorosas reconhecida como a principal causa de dor não dental na região orofacial, afetando os músculos da mastigação, estruturas associadas e articulação temporomandibular, possuindo etiologia complexa e multifatorial. Objetivo: Determinar a prevalência da sintomatologia da disfunção temporomandibular em universitários. Material e método: Trata-se de um estudo do tipo transversal, observacional, com abordagem indutiva, adotando como estratégia de coleta de dados o Questionário de triagem para dor orofacial e desordens temporomandibulares proposto pela Academia Americana de Dor Orofacial. A amostra foi composta de 200 estudantes de Odontologia da Universidade Federal de Campina Grande, Patos, Brasil. Resultados: A maioria da amostra era composta pelo sexo feminino (62,5\%) com idade média de 20,3 anos com desvio padrão de 2,9. Além disso, $60,5 \%$ dos graduandos apresentaram sintomatologia da disfunção. Quando comparados os anos da graduação a maior prevalência ocorreu no $5^{\circ}$ ano $(67,5 \%)$ e o menor para $\circ 2^{\circ}$ ano $(53,8 \%)$. Conclusão: Houve uma alta prevalência dos sinais e sintomas da Disfunção Temporomandibular nesta população, destacando-se a importância da busca detalhada dos sinais e sintomas para a realização da pré-triagem dos pacientes e medidas que possam auxiliar na prevenção e tratamento da DTM.

Descritores: Estudantes; Transtornos da Articulação Temporomandibular; Odontologia.

\section{Abstract}

Introduction: Temporomandibular disorder is a set of painful conditions recognized as the main cause of non-dental pain in the orofacial region, affecting masticatory muscles, associated structures and temporomandibular joint, having a complex and multifactorial etiology. The university population is subject to the development of emotional changes, such as depression and anxiety. These conditions can trigger the onset of TMD symptoms. Objective: To determine the prevalence of symptoms of temporomandibular disorder in university students. Material and method: This is a cross-sectional, observational study with an inductive approach, adopting as a data collection strategy the screening questionnaire for orofacial pain and temporomandibular disorders proposed by the American Academy of Orofacial Pain. The sample consisted of 200 dentistry students from the Federal University of Campina Grande, Patos, Brazil. Results: Most of the sample consisted of women (62.5\%) with a mean age of 20.3 years with a standard deviation of 2.9 . In addition, $60.5 \%$ of the students had symptoms of the dysfunction. When comparing the years of graduation, the highest prevalence occurred in the 5th year (67.5\%) and the lowest for the 2nd year $(53.8 \%)$. Conclusion: There was a high prevalence of the signs and symptoms of Temporomandibular Dysfunction in this population, highlighting the importance of the detailed search for signs and symptoms for the pre-screening of patients and measures that can help in the prevention and treatment of TMD.

Descriptors: Students; Temporomandibular Joint Disorders; Dentistry.

\section{Resumen}

Introducción: El trastorno temporomandibular es un conjunto de condiciones dolorosas reconocidas como la principal causa de dolor no dental en la región orofacial, que afecta a los músculos masticatorios, estructuras asociadas y articulación temporomandibular, de etiología compleja y multifactorial. La población universitaria está sujeta al desarrollo de cambios emocionales, como depresión y ansiedad. Estas condiciones pueden desencadenar la aparición de síntomas de TMD. Objetivo: determinar la prevalencia de síntomas del trastorno temporomandibular en estudiantes universitarios. Material y método: Se trata de un estudio observacional transversal con abordaje inductivo, adoptando como estrategia de recolección de datos el cuestionario de cribado de dolor orofacial y trastornos temporomandibulares propuesto por la Academia Americana de Dolor Orofacial. La muestra estuvo conformada por 200 estudiantes de Odontología de la Universidad Federal de Campina Grande, Patos, Brasil. Resultados: La mayor parte de la muestra estuvo constituida por mujeres (62,5\%) con una edad media de 20,3 años con una desviación estándar de 2,9. Además, el 60,5\% de los estudiantes presentaba síntomas de la disfunción. Al comparar los años de graduación, la mayor prevalencia ocurrió en el $5^{\circ}$ año $(67,5 \%)$ y la más baja en el $2^{\circ}$ año (53,8\%). Conclusión: Existió una alta prevalencia de los signos y síntomas de Disfunción Temporomandibular en esta población, destacando la importancia de la búsqueda detallada de signos y síntomas para el pre-cribado de pacientes y medidas que puedan ayudar en la prevención y tratamiento de TTM.

Descriptores: Estudiantes; Trastornos de la Articulación Temporomandibular; Odontología.

INTRODUÇÃO

$$
\text { Disfunção temporomandibular (DTM) é }
$$

um termo que engloba um conjunto de condições dolorosas crônicas e alterações na região orofacial afetando os músculos da mastigação, as articulações temporomandibulares (ATM) e estruturas associadas $^{1}$. A DTM é reconhecida como a principal causa de dor não dental na região orofacial e afeta negativamente a função do sistema estomatognático, muscular e a qualidade de vida da população em geral ${ }^{2}$. Esse agravo, apresenta etiologia 
complexa e multifatorial, associada a fatores predisponentes, iniciadores e perpetuantes, como alterações oclusais, hábitos parafuncionais, estresse, ansiedade ou anormalidades no disco intra-articular. Tais fatores podem estar relacionados à ocorrência de inflamações articulares, danos e dores musculares ou espasmos ${ }^{3}$. No entanto, a influência desses agentes etiológicos é controversa e ainda não totalmente compreendida 4 .

Os sintomas mais frequentes da DTM envolvem fadiga muscular, ruídos articulares, cefaleia, limitação e/ou desvios dos movimentos da mandíbula e dores na face ${ }^{5-7}$. Os fatores psicossociais estão associados com a gravidade e a persistência dos sintomas clínicos ${ }^{8-10}$. O estresse e a ansiedade, podem causar hiperatividade muscular e o desenvolvimento de hábitos parafuncionais, levando a microtraumas da ATM e lesões musculares ${ }^{11-13}$.

Em função disso, os estudantes universitários são candidatos em potencial para o surgimento de desordens que afetam a ATM, uma vez que os mesmos, no decorrer da graduação, desenvolvem hábitos parafuncionais, tais como apoiar a mão sobre a mandíbula, bruxismo, briquismo, mascar chiclete, morder o lábio, onicofagia e morder objetos $^{13-17}$.

Dessa maneira, o objetivo do trabalho foi avaliar a dor orofacial $e$ as desordens temporomandibulares em estudantes de Odontologia da Universidade Federal de Campina Grande (UFCG) com a finalidade de traçar perfis populacionais, visto que em populações específicas a sintomatologia pode se comportar de maneiras diferentes.

\section{MATERIAL E MÉTODO}

Trata-se de um estudo transversal, observacional, com abordagem indutiva e procedimento comparativo, descritivo, adotando como estratégia de coleta de dados 0 questionário específico. 0 universo foi composto de 387 alunos regularmente matriculados do $1^{\circ}$ ao $10^{\circ}$ período do Curso de Odontologia da Universidade Federal de Campina Grande (UFCG), campus de Patos. O cálculo amostral considerou um grau de confiança de $95 \%$, poder de teste de $50 \%$ e erro aceitável de $5 \%$, obtendo-se uma amostra de 193 participantes.

Para a participação dos graduandos nessa pesquisa foram considerados como critérios de inclusão: estarem regularmente matriculados no Curso de Odontologia e com idade maior que 18 anos. Foram excluídos da pesquisa os graduandos que apresentassem uma ou mais das seguintes características: estudantes em tratamento ortodôntico e/ou que realizaram um procedimento cirúrgico nos últimos 15 dias.

A coleta de dados foi realizada entre os meses de setembro do ano 2019 e março do ano 2020 por um único pesquisador através do questionário de triagem para dor orofacial e desordens temporomandibulares recomendado pela Academia Americana de Dor Orofacial (American Academy Of Orofacial Pain), sendo composto por dez perguntas, com respostas sim/não, a sinais e sintomas mais frequentes de dor orofacial e DTM. Os dados foram coletados durante o intervalo entre as aulas da graduação, com aplicação rápida levando em torno de 8 minutos, podendo ser aplicado individualmente. Além disso, também foi avaliado a presença de pelo menos uma das sintomatologias, uma vez que o participante da pesquisa tendo marcado "sim" para uma das perguntas do questionário já caracterizaria presença de sintomatologia DTM ou "não" à total ausência delas em cada estudante.

Após coletados, os dados foram registrados na forma de banco de dados do programa de informática em planilha do Microsoft Excel®. Após a análise dos questionários pode-se construir gráficos com os valores encontrados para cada variável presente no Adobe Illustrator.

A execução da pesquisa foi autorizada pelo Comitê de Ética em Pesquisa (CEP), apresentando CAAE 13834919.5.0000.5181, todos os sujeitos em pesquisa tiveram acesso ao termo de consentimento livre e esclarecido (TCLE) e participaram da pesquisa de forma espontânea.

RESULTADOS

A amostra total foi composta por 200 graduandos com idade variando entre 18 e 39 anos com média de 20,3 anos com desvio padrão de 2,9, sendo $62,5 \%$ (125) do sexo feminino e $37,5 \%$ (75) do sexo masculino. Após a análise dos questionários pôde-se construir gráficos com os valores encontrados para cada variável presente. Os acadêmicos estão distribuídos de acordo com ano do curso, sexo e resposta afirmativas ou negativas.

As Figuras 1 e 2 demonstram a frequência absoluta para as variáveis dificuldade, dor ou ambas, ao realizar o movimento de abertura bucal e a presença de travamento ou deslocamento da mandíbula. As Figuras 3 e 4 apresentam os resultados para os questionamentos sobre dificuldade, dor ou 
ambas ao mastigar, falar ou usar os maxilares e a percepção de ruídos articulares.

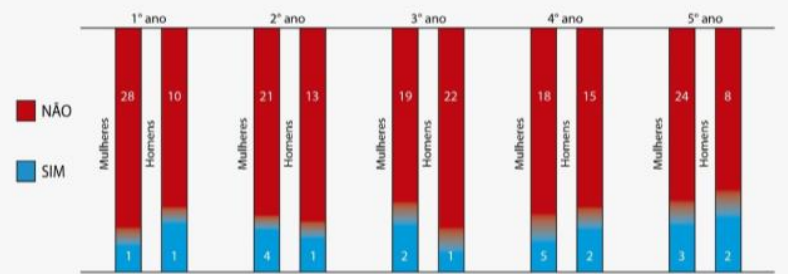

Figura 1: Frequência absoluta para dificuldade, dor ou ambas ao abrir a boca.

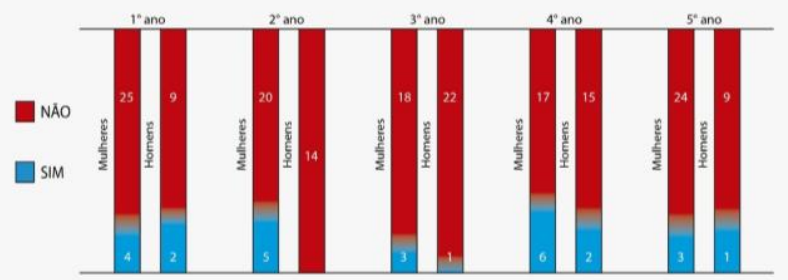

Figura 2: Frequência absoluta para mandíbula fica "presa", "travada" ou sai do lugar.

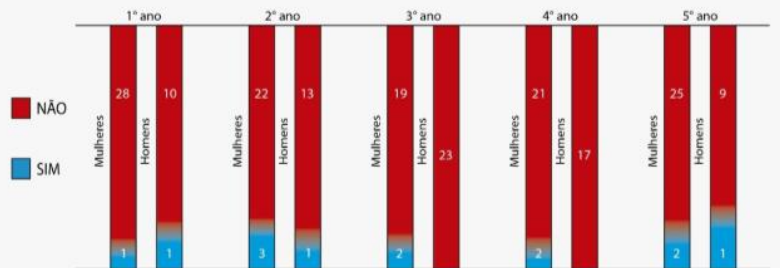

Figura 3: Frequência absoluta para dificuldade, dor ou ambas ao mastigar, falar ou usar seus maxilares.

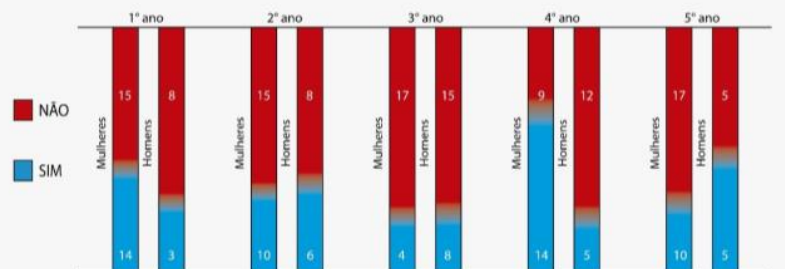

Figura 4: Frequência absoluta para ruídos nas articulações dos maxilares.

As Figuras 5 e 6 apresentam, respectivamente, os dados para as variáveis sensação de rigidez, apertamento e cansaço nos maxilares e dor nas orelhas, têmporas e bochechas.

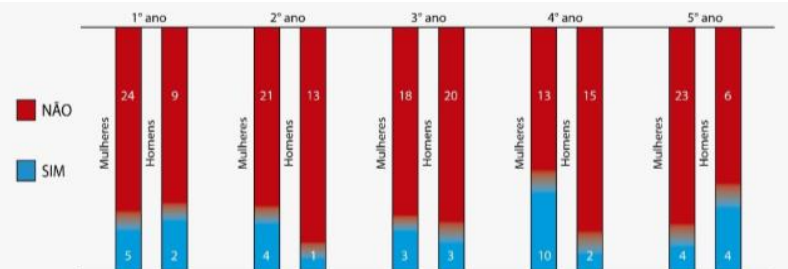

Figura 5: Frequência absoluta para maxilares rígidos, apertados ou cansados.

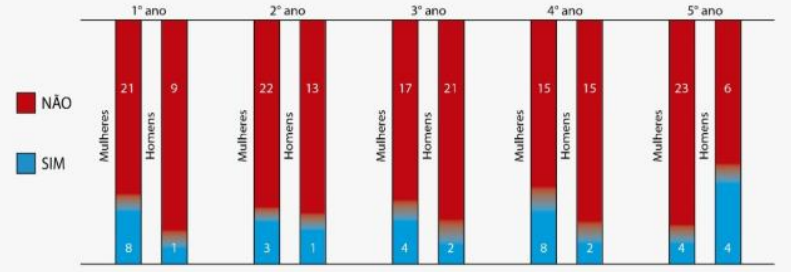

Figura 6: Frequência absoluta para dor nas orelhas ou em volta delas, têmporas e bochechas.

Os dados obtidos, respectivamente, para as variáveis cefaleia, dor cervical ou dentária frequentes além da presença de trauma recente na cabeça, pescoço ou maxilares estão apresentados nas Figuras 7 e 8.

$E$ as Figuras 9 e o 10 demonstram a frequência absoluta encontrada para as variáveis alteração recente na mordida e realização de um tratamento para um problema não explicado na ATM, respectivamente.

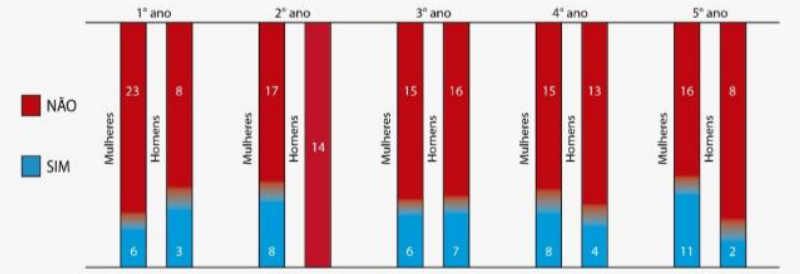

Figura 7: Frequência absoluta para cefaleia, dor no pescoço ou nos dentes.

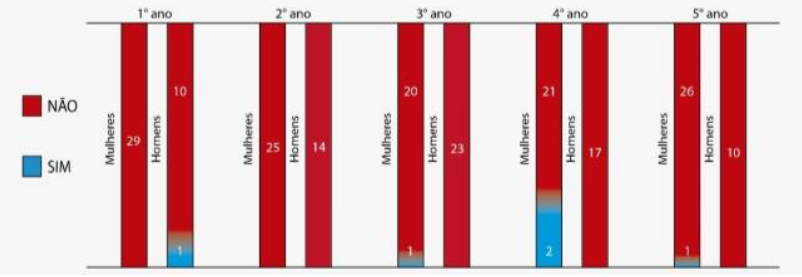

Figura 8: Frequência absoluta para trauma recente na cabeça, pescoço ou maxilares.

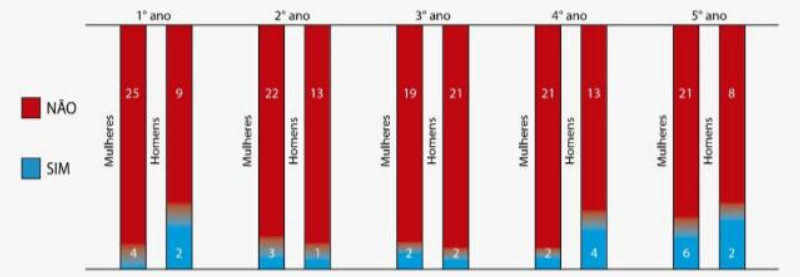

Figura 9: Frequência absoluta para alguma alteração recente na mordida.

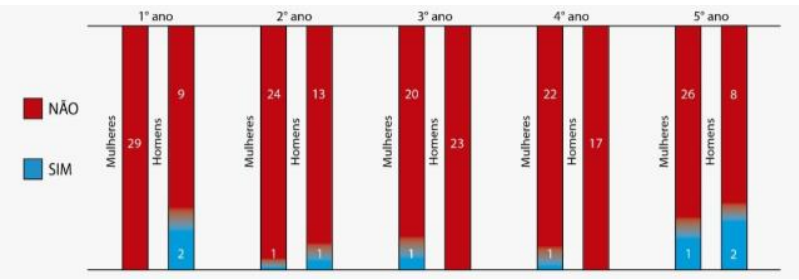

Figura 10: Frequência absoluta para realização de tratamento para um problema não explicado na ATM.

Quando avaliado os questionários de forma individual, observando as 10 perguntas respondidas, foi observado um maior número de estudantes com sintomatologia da DTM. Já que $60,5 \%$ (121) dos entrevistados responderam "sim" para pelo menos uma das questões presentes. Em relação ao comportamento entre os sexos, há uma maior prevalência da sintomatologia nas mulheres, onde 63,2\% (79) delas assinalaram "sim" em pelo menos uma das perguntas. Em contrapartida, o sexo masculino apresentou percentual de 56\% (42) que afirmaram apresentar pelo menos um sintoma presente no questionário. 
Com relação aos sintomas, o mais prevalente foi a percepção dos ruídos na articulação dos maxilares, visto que 79 (39,5\%) alunos responderam sim para pergunta número 4. Em contrapartida, o menos frequente foi aquele relacionado à questão 8 , onde apenas 5 pessoas afirmaram ter sofrido algum trauma recente na cabeça, pescoço ou maxilares. Em relação ao ano de curso, no $5^{\circ}$ ano $67,5 \%$ (25) dos estudantes apresentaram pelo menos uma sintomatologia e $5(13,5 \%)$ assinalaram mais da metade dos sintomas listados.

DISCUSSÃO

A DTM apresenta uma etiologia complexa e multifatorial caracterizada por condições que afetam as regiões musculoesqueléticas e neuromusculares que se relacionam com a $\mathrm{ATM}^{3}$. Por consequência, o diagnóstico precoce utiliza a identificação de sinais e sintomas como uma importante ferramenta. Nesse contexto, a literatura tem defendido o uso de questionários validados com fácil aplicação e interpretação com o objetivo na uniformização da comparação de dados ${ }^{18,19,20}$.

Neste estudo observou-se que uma considerável parcela dos estudantes universitários apresentara sintomatologia de DTM $(60,5 \%)$, corroborando com os resultados encontrados nos estudos de Augusto et al. ${ }^{21}$ que obtiveram prevalência dos sinais e sintomas $(71,9 \%)$ nos universitários dos cursos de ciências da saúde avaliados através do índice anamnésico de fonseca. Além disso, foram encontrados no estudo Bezerra et al. ${ }^{22}$, que avaliaram a prevalência de DTM e o grau de ansiedade em 336 estudantes universitários, uma prevalência de $62,5 \%$ dos sinais e sintomas de DTM, bem como na pesquisa de Oliveira et al. ${ }^{23} \mathrm{com}$ prevalência da DTM em $68,6 \%$, que avaliaram estudantes de diversas regiões do Brasil.

A população universitária é propensa ao desenvolvimento de alterações emocionais, como depressão e ansiedade ${ }^{24-26}$. Essas condições podem desencadear o aparecimento de hábitos parafuncionais e espasmos nos músculos mastigatórios sobrecarregando 0 funcionamento da $A_{T M}{ }^{27,28}$. o que pode justificar a alta prevalência de tais sintomatologias nesta pesquisa.

O presente estudo demonstrou uma prevalência da sintomatologia no sexo feminino $(63,2 \%)$, corroborando com outras pesquisas que obtiveram resultados semelhantes tais como Bezerra et al. ${ }^{22}$ que $65,3 \%$ do sexo feminino apresentaram DTM e Guerra et al. ${ }^{29}$ que encontraram prevalência no sexo feminino de $63,5 \%$. Este fato pode estar relacionado às diferenças fisiológicas entre os sexos, onde a mulher apresenta maiores patologias ósseas e psicossociais comparadas ao homem ${ }^{30}$. Ademais, outra possível justificativa é que a produção de estrógeno pode estar relacionada com a maior prevalência de sinais e sintomas da DTM em mulheres na idade reprodutiva, já que este hormônio é responsável pela regulação do crescimento e desenvolvimento ósseo, influenciando no mecanismo central e periférico da dor ${ }^{31}$.

Quanto a alta prevalência dos sinais e sintomas de DTM em estudantes do último ano da faculdade quando comparado aos acadêmicos de outros períodos, é possível que haja uma relação com maior nível de estresse presente neste ano da graduação devido ao acúmulo de atividades, que demandam grande dedicação do estudante, além da produção do trabalho de conclusão de curso, da expectativa com a formatura e com a inserção no mercado de trabalho. Augusto et al.21 (2016) afirma que as constantes avaliações do curso, o tempo limitado para realização de tarefas diárias e o tempo reduzido para as relações interpessoais e intrapessoais contribuem como fatores para um alto nível de estresse.

De sintomatologia variada, a Disfunção Temporomandibular apresenta patogênese mal compreendida, de difícil diagnóstico e controle, sendo importante identificar os sinais e sintomas previamente e os corretos fatores etiológicos envolvidos32. Nos participantes desta pesquisa, a sintomatologia mais comum foi a percepção dos ruídos na articulação dos maxilares, em contrapartida o trauma recente na cabeça, pescoço ou maxilares foi pouco relatado.

A DTM é considerada a maior causa de dor não dentária na região orofacial, afetando negativamente a qualidade de vida e prejudicando o funcionamento social, assim como o bem-estar físico e psicológico do paciente ${ }^{1,33,34}$, assim como foi visto neste estudo, em que outros sintomas, com alta frequência de respostas afirmativas, foram aqueles relacionados a dores no pescoço, nos dentes, nas orelhas, nas têmporas, nas bochecha e cefaleia.

Dessa forma, observa-se a importância da anamnese para coleta de dados sintomatológicos da doença e o estudo de cada caso para melhor adequar a técnica de tratamento a ser utilizada. Há a necessidade de avaliações clínicas multidisciplinares nos indivíduos identificados com DTM para que o tratamento seja otimizado, minimizando a morbidade e diminuindo os custos e o número 
de consultas prestadas aos pacientes ${ }^{34,35}$.

Os resultados obtidos neste trabalho demonstraram que a aplicação do questionário foi eficaz e útil para a pré-triagem de pacientes com DTM e para o levantamento da sintomatologia. No entanto, não deve ser a única ferramenta para o diagnóstico da disfunção sendo necessário um exame clínico detalhado, como afirmam Manfredi et al. ${ }^{36}$.

CONCLUSÃO

Diante do exposto, pode-se concluir que houve uma alta prevalência de sintomatologia da Disfunção Temporomandibular nesta população, destacando a importância da busca detalhada dos sinais e sintomas para a realização da pré-triagem dos pacientes antes da realização do exame clínico. A aplicação do questionário mostrou-se eficaz para 0 levantamento da sintomatologia, entretanto não deve ser a única ferramenta para diagnóstico de DTM.

\section{REFERÊNCIAS}

1. Al-Khotani A, Naimi-Akbar A, Albadawi E, Ernberg M, Hedenberg-Magnusson $B$, Christidis N. Prevalence of diagnosed temporomandibular disorders among Saudi Arabian children and adolescents. J Headache Pain. 2016;17(1):41.

2. Giro G, Policastro VB, Scavassin PM, Leite AR, Mendoza Marin DO, Gonçalves DA, et al. Mandibular kinesiographic pattern of women with chronic TMD after management with educational and self-care therapies: A doubleblind, randomized clinical trial. The Journal of prosthetic dentistry. 2019;116(5):749-55.

3. Buescher JJ. Temporomandibular joint disorders. Am Fam Physician. 2007;76(10):1477-82.

4. Paulino MR, Moreira VG, Lemos GA, Silva PLPD, Bonan PRF, Batista AUD. Prevalência de sinais e sintomas de disfunção temporomandibular em estudantes prévestibulandos: associação de fatores emocionais, hábitos parafuncionais e impacto na qualidade de vida. Ciênc Saúde Coletiva. 2018;23:173-86.

5. Donnarumma MC, Muzilli CA, Ferreira C, Nemr K. Temporomandibular Disorders: signs, symptoms and multidisciplinary approach. Rev CEFAC. 2010;12(5):788-94

6. Dantas AM, Santos EJ, Vilela RM, Lucena LB. Perfil epidemiológico de pacientes atendidos em um serviço de controle da dor orofacial. Rev Odontol UNESP. 2015;44(6):313-19.

7. Garbelotti TO, Turci AM, Serigato JM, Pizzol K, Franco-Micheloni AL. Effectiveness of acupuncture for temporomandibular disorders and associated symptoms. Rev Dor. 2016;17(3):223-27.

8. Conti PC, Pinto-Fiamengui LM, Cunha CO, Conti AC. Orofacial pain and temporomandibular disorders: the impact on oral health and quality of life. Braz Oral Res. 2012;26(Suppl 1):120-23

9. Nishiyama A, Kino K, Sugisaki M, Tsukagoshi $\mathrm{K}$. Influence of Psychosocial Factors and Habitual Behavior in Temporomandibular Disorder-Related Symptoms in a Working Population in Japan. Open Dent J. 2012;6: 240-47

10. Piccin CF, Pozzebon D, Chiodelli L, Boufleus J Pasinato F, Corrêa EC. Aspectos clínicos e psicossociais avaliados por critérios de diagnóstico para disfunção temporomandibular. Rev CEFAC. 2016;18(1):113-19.

11. Winocur E, Gavish A, Finkelstein T, Halachmi M, Gazit E. Oral habits among adolescent girls and their association with symptoms of TMD. J Oral Reabil. 2001;28(7):624-29.

12. Carvalho LPMD, Piva MR, Santos TDS, Ribeiro CF, Araújo CRFD, Souza LBD. Estadiamento clínico da disfunção temporomandibular: estudo de 30 casos. Odontol clín.-cient. 2008;7(1): 47-52.

13. Medeiros SP, Batista AUD, Forte FDS. Prevalência de sintomas de disfunção temporomandibular e hábitos parafuncionais em estudantes universitários. RGO Rev Gaúch Odontol. 2011;59(2):201-18.

14. Alves-Rezende MCR, Silva JS, Soares BS, Bertoz FA, Oliveira DTN, Alves-Claro APR. Estudo da prevalência de sintomatologia temporomandibular em universitários brasileiros de Odontologia. Rev Odontol Araçatuba. 2009;30(1):9-14

15. Barbosa JA, Swerts AA. Prevalência da disfunção temporomandibular em graduandos do curso de Odontologia da Universidade José de Rosário Vellano - UNIFENAS, MG. Rev CROMG. 2011;12(2):65-8.

16. Melchior MO, Mazzetto MO, Felício CM. Temporomandibular disorders and parafunctional oral habits: an anamnestic study. Dental Press J Orthod. 2012;17(2):83-9.

17. Pinto RG, Leite WM, Sampaio LS, Sanchez MO. Association between temporomandibular signs and symptoms and depression in undergraduate students: descriptive study. Rev Dor. 2017;18(3):217-24.

18. Fonseca DM, Bonfante G, Valle AL, Freitas SFT. Diagnóstico pela anamnese da disfunção craniomandibular.RGO.1994;42(1):23-8.

19. Feteih RM. Signs and symptoms of temporomandibular disorders and oral parafunctions in urban Saudi Arabian adolescents: a research report. Head Face Med. 2006;2:25. 
20. Ichesco E, Quintero A, Clauw DJ, Scott P, Pia S, Geoffrey EG, et al. Altered functional connectivity between the insula and the cingulated cortex in patients with temporomandibular disorder: a pilot study. Headache. 2012;52(3):441-54.

21. Augusto VG, Perina KCB, Penha DSG, Santos DCAD, Oliveira VAS. Temporomandibular dysfunction, stress and common mental disorder in university students. Acta Ortop Bras. 2016;24(6):330-33.

22. Bezerra BPN, Ribeiro AIAM, Farias ABL, Farias ABLF, Fontes LBC, Nascimento SR et al. Prevalência da disfunção temporomandibular e de diferentes níveis de ansiedade em estudantes universitários. Rev DOR. 2012;13(3):235-42

23. Oliveira AS, Grossi DB, Dias EM. Sinais e sintomas de disfunção temporomandibular nas diferentes regiões brasileiras. Fisioter Pesq. 2008;15(4):392-97.

24. Soares LFF, Coelho LM, Moreno A, Almeida DAF, Haddad MF. Ansiedade e depressão associados à dor e desconforto das desordens temporomandibulares. BrJP. 2020;3(2):147-52.

25. Turner K, McCarthy VL. Stress and anxiety among nursing students: a review of intervention strategies in literature between 2009 and 2015. Nurse Educ Pract. 2017;22:21-9

26. Haidar SA, de Vries NK, Karavetian M, El-Rassi R. Stress, anxiety, and weight gain among university and college students: a systematic review. J Acad Nutr Diet. 2018;118(2):261-74

27. Minghelli B, Morgado M, Caro T. Association of temporomandibular disorder symptoms with anxiety and depression in Portuguese college students. J Oral Sci. 2014;56(2):127-33

28. Paiva RC. A depressão e a disfunção temporomandibular - estudo clínico [dissertação]. Viseu: Universidade Católica Portuguesa; 2012.

29. Guerra C, Pinho IBS, Oliveira LTGC, Nicésio PHA, Rocha PBVF, Gomes JSR et al. Prevalência de sinais e sintomas da disfunção temporomandibular e sua relação com fatores psicológicos em acadêmicos de medicina de barbacena. Rev Interdisciplinar de Estudos Experimentais Animais e Humanos 2018; 10.Único.

30. Kostrzewa-Janicka J, Pietrzak B, Jurkowski P, Wielgos M, Binkowska M, MierzwinskaNastalska E. Effects of oral contraceptives on the treatment for internal derangements in temporomandibular joints in women. Neuro Endocrinol Lett. 2013;34(6):566-72.

31. Kim TY, Shin JS, Lee J, Lee YJ, Kim MR, Ahn $Y J$ et al. Gender difference in associations between chronic temporomandibular disorders and general quality of life in Koreans: a cross sectional study. PLoSOne.2015;10(12): e0145002.

32. Chisnoiu AM, Picos AM, Popa S, Chisnoiu PD, Lascu L, Picos A et al. Factors involved in the etiology of temporomandibular disorders - a literature review. Clujul Med. 2015;88(4):473-8.

33. Souza Barbosa T, Gaviao MB, Castelo PM, Leme MS. Factors Associated with Oral Healthrelated Quality of Life in Children and Preadolescents: A Cross-sectional Study. Oral Health Prev Dent. 2016;14(2):137-49.

34. Sipilä K, Tolvanen M, Mitrirattanakul S, Sitthisomwong $P$, Järvelin MR, Taanila $A$, et al. Orofacial pain and symptoms of temporomandibular disorders in Finnish and Thai populations. Acta Odontol Scand. 2015; 73(5):330-35

35. Cruz JHA, Sousa LX, Oliveira BF, Andrade júnior $\mathrm{FP}$, Alves ASG, Oliveira Filho AA. Disfunção Tempormandibular: Revisão sistematizada. Arch Healt Invest. 2020; $9(6): 555-60$

36. Manfredi APS, Silva AA, Vendite L. Avaliação da sensibilidade do questionário de triagem para dor orofacial e desordens temporomandibulares recomendado pela Academia Americana de Dor Orofacial. Rev Bras Otorrinolaringol. 2001;67(6):763-68.

\section{CONFLITO DE INTERESSES}

Os autores declaram não haver conflitos de interesse

\section{AUTOR PARA CORRESPONDÊNCIA}

\section{José Henrique de Araújo Cruz}

Rua Paulo Diogenes, número 57, Centro, 59990 - 000 Rafael Fernandes - RN, Brasil Telefone: (83) 99625-0125.

E-mail: henrique_araujo1992@hotmail.com
Submetido em 18/11/2020 Aceito em 30/04/2021 